\title{
Association between severity of disease and thoracic wall dimensions among chronic obstructive pulmonary disease patients - A cross sectional study
}

\author{
Viswambhar Vallabhaneni' ${ }^{1}$ Bandlamudi Sowjanya ${ }^{2}$, Aruna Shanmuganathan ${ }^{3}$, Meenakshi Narasimhan ${ }^{4}$, Ashwin \\ Kailash Jayakumaran ${ }^{5}$, Navaneetham Karunakaran ${ }^{6}$

\begin{abstract}
${ }^{1}$ Associate Professor and in charge, ${ }^{6}$ Respiratory Therapist, Department of Respiratory Medicine, Shri Sathya Sai Medical College and Research Institute Ammapettai, Kanchipuram, District-603103, Tamil Nadu, India

${ }^{2}$ Associate Professor, Department of Anatomy, ${ }^{3}$ Professor, ${ }^{4}$ Professor and Head, Kanchipuram District-603103, Tamil Nadu, India
\end{abstract} \\ ${ }^{5}$ Second Year PG Student, Department of Respiratory Medicine, Chettinad Hospital and Research Institute, Kelambakkam,
}

(Received: March 2020

Revised: July 2020

Accepted: August 2020)

Corresponding author: Bandlamudi Sowjanya. Email: sowjanya.vneni@gmail.com

\begin{abstract}
Introduction and Aim: Pulmonary function worsens with the intensification of disease severity. Chronic obstructive pulmonary disease (COPD) may cause changes in the shape of the thoracic cage due to increasing lung volumes and dynamic hyperinflation. To assess the association between the severity of COPD and thoracic wall dimensions among COPD patients.
\end{abstract}

Materials and Methods: A cross-sectional study was conducted in a tertiary care teaching hospital on 80 COPD subjects aged above 18 years. Based on the severity of obstruction of COPD, subjects were divided into two groups-moderate obstruction and severe obstruction. Severity of obstruction constituted the primary outcome variable. The primary explanatory variables were the dimensions of the thoracic wall. Their association was assessed by calculating the Spearman correlation and Pearson correlation coefficient and represented in a scatter diagram.

Results: Majority (60\%) had an MMRC dyspnea grading of 3. Moderate Obstruction was seen in $62.5 \%$ of subjects while $37.50 \%$ had severe obstruction. There was no statistically significant difference between the groups with respect to antero posterior (AP) diameter, transverse diameter, AP to transverse diameter ratio and Height of Diaphragm (HDI). There was a moderate negative correlation FVC (L) and HDI, FEV1/FVC (\%) and HDI which was statistically significant. There was also a weak negative statistically significant correlation between FVC (L) and AP diameter, FVC (L) and transverse Diameter, FVC (L) and AP to transverse diameter ratio, FEV1/FVC (\%) and AP diameter, FEV1/FVC (\%) and transverse diameter.

Conclusion: There was a significant moderate negative correlation between the height of the diaphragm and severity of COPD (FVC, FEV1/FVC \%). In COPD, the mechanical deformation leads to difficulty in breathing because of reduction in the lung capacity of patients. Early and aggressive treatment in patients with COPD can be beneficial.

Keywords: Chronic obstructive pulmonary disease; global initiative for chronic obstructive lung disease; medical research council dyspnoea scale; barrel-shaped chest; spirometry, diaphragm.

\section{INTRODUCTION}

$\mathrm{C}$ hronic obstructive pulmonary disease (COPD) is characterized by progressive, irreversible airflow obstruction, and enhanced chronic inflammatory responses to noxious particles or gases in the airways and lungs (1). The Global Initiative for Chronic Obstructive Lung Disease (GOLD) was initiated in 1998 to improve diagnostic and treatment outcomes and is updated yearly (2). GOLD reports are considered to be essential evidence-based reference tools for the implementation of effective management plans and represent the current best practices for the care of patients with COPD (3). Based on spirometry results, four grades of COPD have been described according to GOLD criteriaMild, Moderate, Severe and Very severe disease. Multidimensional GOLD classification, takes into account both exacerbation risk and symptom assessment. High risk will include COPD GOLD 3 or 4 (severe or very severe) or a history of $\geqslant 2$ exacerbations in a year. The modified Medical Research Council (mMRC) scale $\geqslant 2$ indicates more symptoms. In a significant proportion of patients with COPD, reduced lung, elastic recoil along with limitation in expiratory flow eventually leads to hyperinflation. COPD patients may exhibit increased anteroposterior (AP) diameter, leading to a "barrelchest". Patients with COPD exhibited an increased AP diameter of the thoracic cage compared with normal controls (4). Lung function deteriorates with increase in disease severity. Parenchymal attenuation parameters were significantly worse in patients with higher GOLD stages (5). The diaphragm also tends to be shortened in subjects with COPD due to pulmonary hyperinflation (6). In a highly populated developing country like India, there is a paucity of knowledge with regards to the association between 
thoracic dimensions and severity of COPD. The severity of COPD is mainly defined on the basis of forced expiratory volume in 1 second $\left(\mathrm{FEV}_{1}\right)$. However, it does not adequately reflect all the factors needed to be taken into account in its management. Treatment with pharmacological agents is based on the level of symptoms and the risk of exacerbations. The mMRC dyspnea scale is an established simple questionnaire for quantifying disability associated with dyspnea used in clinical practice (7).

Destruction of lung parenchyma and small airway disease are major components of air trapping and airflow limitation in COPD. Hence, we assessed the association between the severity of COPD and thoracic wall dimensions among COPD patients.

\section{MATERIAL AND METHODS}

A cross-sectional study was conducted in the department of pulmonary medicine of a tertiary care rural teaching hospital in Chennai, India between January to December 2019 on 80 COPD subjects aged above 18 years, with disease duration of a minimum of 5 years. The study subjects were included based on the clinical history and pulmonary function testing (FEV1/Forced Vital Capacity (FVC) value $<70 \%$ ) as per the GOLD 2019 guidelines in table 1. $(2,3)$ Patients with a history of Tuberculosis or major trauma to the chest wall were excluded.

Table 1: GOLD staging $(2,3)$ (in patients with FEV1/FVC < 0.70)

\begin{tabular}{|c|c|}
\hline STAGE & Post bronchodilator FEV-1 values \\
\hline I & $\geq 80 \%$ \\
\hline II & $50-79 \%$ \\
\hline III & $30-49 \%$ \\
\hline IV & $\leq 30 \%$ \\
\hline
\end{tabular}

The study was approved by the Institutional human ethics committee (Ref: IHEC.No.04/18/dec.2015). Informed written consent was obtained from all the participants included in the study. Confidentiality was maintained throughout the study period. The severity of COPD was considered as the primary outcome variable. The Primary explanatory variables were the dimensions of the thoracic wall. Data was entered in Microsoft excel and analysis was carried out with IBM SPSS version 22. P-value < 0.05 was considered statistically significant. Descriptive analysis was carried out by mean and standard deviation for quantitative variables, frequency, and proportion for categorical variables. Data was also represented using appropriate diagrams. All Quantitative variables were checked for normal distribution within each category of the explanatory variable by using visual inspection of histograms and normality Q-Q plots. Categorical outcomes were compared between study groups using Chi-square test /Fisher's Exact test (If the overall sample size was $<20$ or if the expected number in any one of the cells is $<5$, Fisher's exact test was used). For normally distributed Quantitative parameters, the mean values were compared between study groups using Independent sample t-test. Association between quantitative explanatory and outcome variables was assessed by calculating Spearman correlation and Pearson correlation coefficient and the data was represented in a scatter diagram. Linear regression was performed to assess the outcome parameter using the explanatory variables by forming the linear regression equation.

\section{RESULTS}

A total of 80 subjects were included in the final analysis. Among the study population, $17(21.25 \%)$ participants belong to MMRC Grade II, 48 (60.00\%) participants belong to MMRC Grade III and 15 (18.75\%) participants belong to MMRC Grade IV. Among the study population, 50 (62.50\%) participants belong to Moderate Obstruction and 30 $(37.50 \%)$ participants belong to Severe Obstruction.

Table 2: Descriptive analysis of MMRC in the study population $(\mathrm{N}=80)$

\begin{tabular}{|c|c|c|}
\hline Parameter & Frequency & Percentages \\
\hline \multicolumn{3}{|c|}{ MMRC } \\
\hline Grade II & 17 & $21.25 \%$ \\
\hline Grade III & 48 & $60.00 \%$ \\
\hline Grade IV & 15 & $18.75 \%$ \\
\hline \multicolumn{2}{|c|}{ Severity of obstruction } \\
\hline Moderate Obstruction & 50 & $62.50 \%$ \\
\hline Severe Obstruction & 30 & $37.50 \%$ \\
\hline
\end{tabular}

The mean age was $60.52 \pm 3.78$ in Moderate Obstruction and it was 59.23 \pm 3.11 in Severe Obstruction, and the difference between the two groups was statistically not significant (P-value 0.120). out of 50 participants in Moderate Obstruction, 36 (72\%) participants were male and 14 (28\%) participants were female, out of 30 participants in Severe Obstruction, 26 (86.67\%) participants were male and $4(13.33 \%)$ participants were female, the difference in the 2 groups was statistically not significant (P-value 0.128). The mean Body Mass Index (BMI) was 24.56 \pm 4.73 in Moderate Obstruction and it was $27.03 \pm 5.26$ in Severe Obstruction, and the difference between the two groups was statistically significant (P-value $0.033)$. The mean of AP Diameter (mm) was $10.53 \pm$ 1.71 in Moderate Obstruction and it was $12.16 \pm 1.55$ in Severe Obstruction, and the difference between 2 groups was statistically significant (P-value $<0.001$ ). The mean of Transverse Diameter $(\mathrm{cm})$ was $22.65 \pm$ 1.79 in Moderate Obstruction and it was $22.58 \pm 2.06$ in Severe Obstruction, and the difference between 2 groups was statistically not significant (P-value $0.882)$. The mean of Height of Diaphragm $(\mathrm{cm})$ was $23.28 \pm 2.59$ in Moderate Obstruction and it was $23.47 \pm 2.66$ in Severe Obstruction, and the difference between 2 groups was statistically not significant (P-value 0.757). The mean of AP to Transverse diameter ratio was $0.47 \pm 0.08$ in 
Moderate Obstruction and it was $0.55 \pm 0.05$ in Severe Obstruction, and the difference between 2

groups was statistically significant $(\mathrm{P}-\mathrm{value}<0.001)$.

Table 3: Comparison of mean of age, BMI between Interpretation $(\mathrm{N}=80)$

\begin{tabular}{|c|c|c|c|}
\hline \multirow[b]{2}{*}{ Parameter } & \multicolumn{2}{|c|}{ Severity of obstruction } & \multirow[b]{2}{*}{ P-value } \\
\hline & $\begin{array}{c}\text { Moderate } \\
\text { Obstruction }(\mathrm{N}=\mathbf{5 0})\end{array}$ & $\begin{array}{c}\text { Severe } \\
\text { Obstruction }(\mathrm{N}=\mathbf{3 0})\end{array}$ & \\
\hline AGE (Mean \pm SD) & $60.52 \pm 3.78$ & $59.23 \pm 3.11$ & 0.120 \\
\hline \multicolumn{4}{|c|}{ Gender (n \%) } \\
\hline Male & $36(72 \%)$ & $26(86.67 \%)$ & \multirow{2}{*}{0.128} \\
\hline Female & $14(28 \%)$ & $4(13.33 \%)$ & \\
\hline BMI & $24.56 \pm 4.73$ & $27.03 \pm 5.26$ & 0.033 \\
\hline AP diameter (Mean \pm SD) & $10.53 \pm 1.71$ & $12.16 \pm 1.55$ & $<0.001$ \\
\hline Transverse Diameter (Mean \pm SD) & $22.65 \pm 1.79$ & $22.58 \pm 2.06$ & 0.882 \\
\hline Height of Diaphragm $(\mathrm{cm})($ Mean \pm SD) & $23.28 \pm 2.59$ & $23.47 \pm 2.66$ & 0.757 \\
\hline $\mathrm{AP}$ to transverse diameter ratio (Mean $\pm \mathrm{SD})$ & $0.47 \pm 0.08$ & $0.55 \pm 0.05$ & $<0.001$ \\
\hline
\end{tabular}

There was a weak negative correlation between FVC (L) and AP Diameter (mm) (r Value: -0.326, P-value: $0.003)$. There was a weak negative correlation between FVC (L) and Transverse Diameter $(\mathrm{cm})(\mathrm{r}$ Value: -0.092, P-value: 0.415). There was a weak positive correlation between FVC (L) and Height of Diaphragm (cm) (r Value: 0.013, P-value: 0.912). There was a weak negative correlation between FVC (L) and AP to transfer the Transverse diameter ratio (r Value: -0.330, P-value: 0.003).
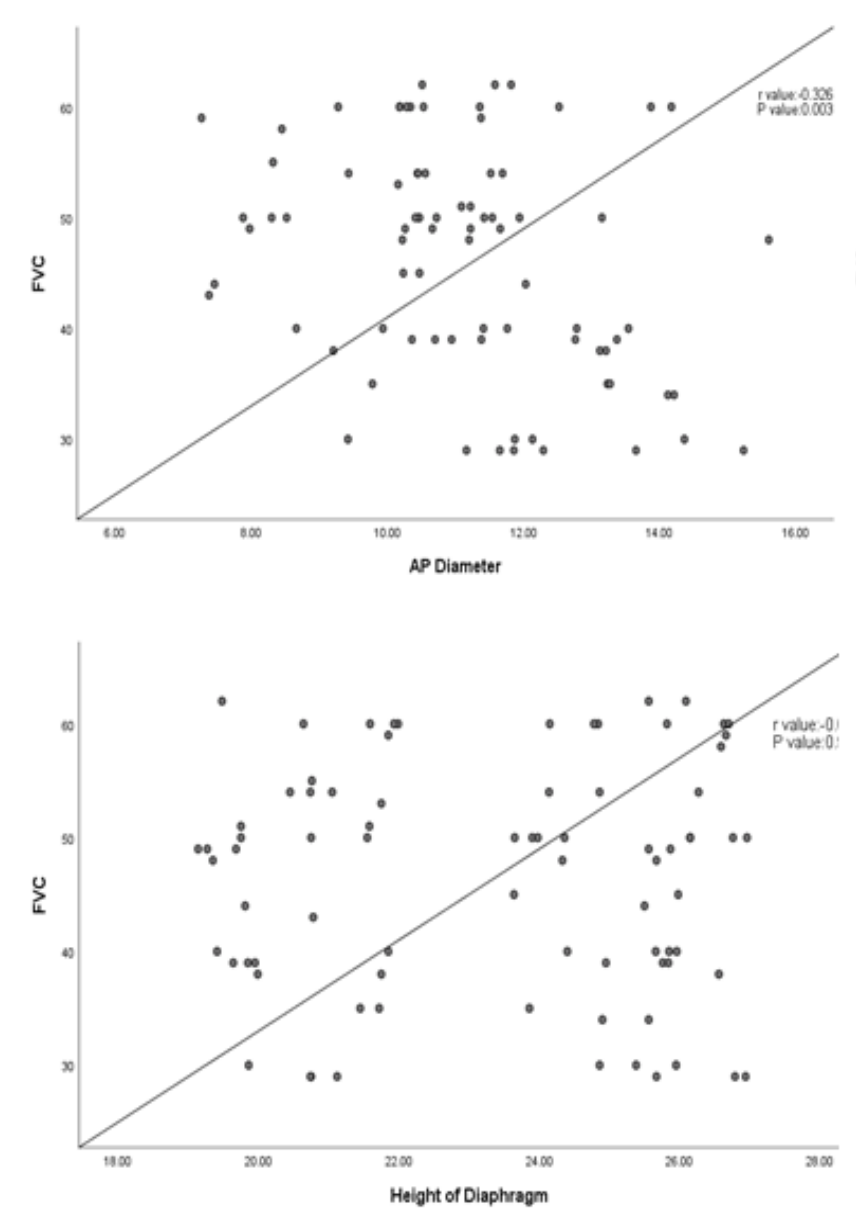

There was a weak negative correlation between FEV1/FVC (\%) and AP Diameter (mm) (r Value: 0.328 , P-value: $<0.001)$. There was a weak negative correlation between FEV1/FVC (\%) and Transverse Diameter (cm) (r Value: -0.095, P-value: 0.400). There was a weak negative correlation between FEV1/FVC (\%) and Height of Diaphragm $(\mathrm{cm})(\mathrm{r}$ Value: -0.023, P-value: 0.840). There was a weak negative correlation between FEV1/FVC (\%) and AP to Transverse diameter ratio ( $\mathrm{r}$ Value: -0.274 , Pvalue: 0.014 ).
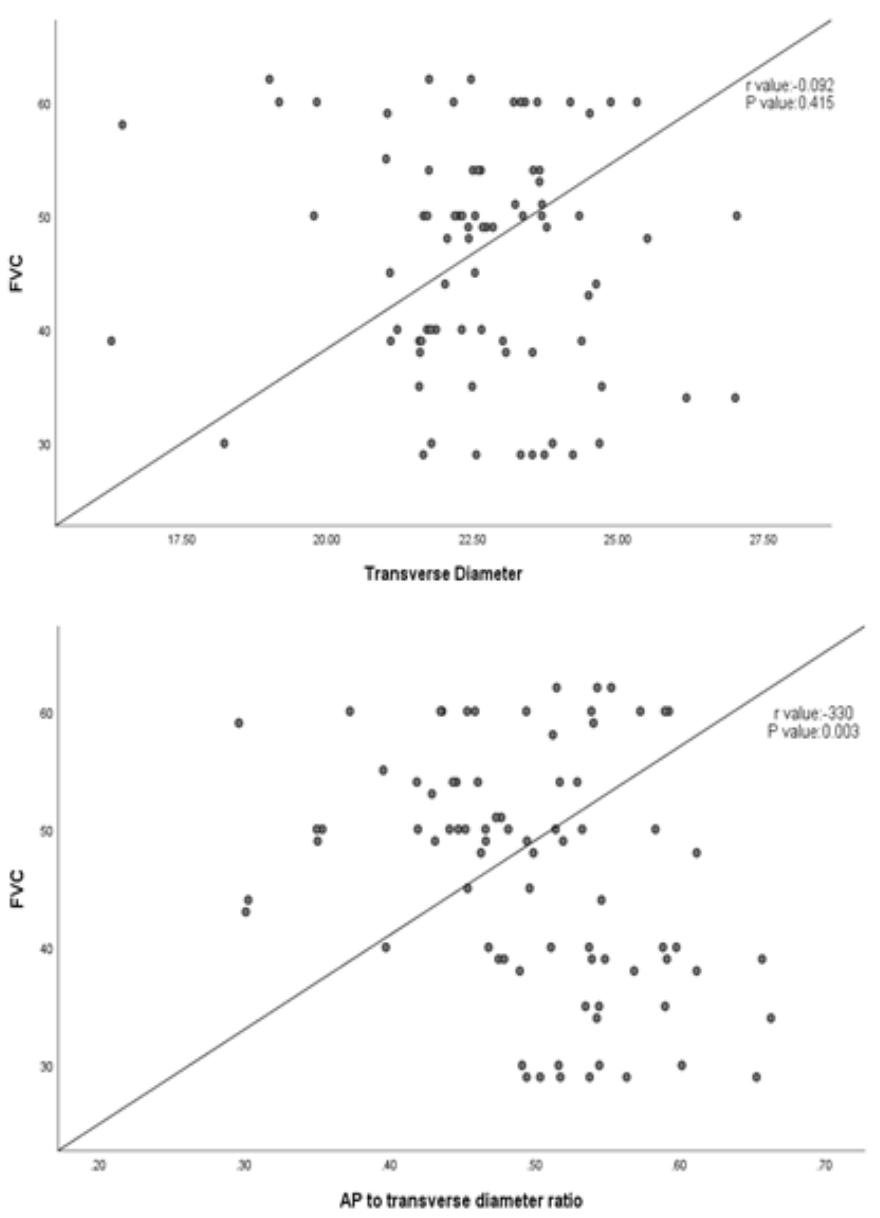

Fig. 1: Scatter plot for Correlation between Thoracic wall dimensions and FVC (L) in the study population $(\mathrm{N}=80)$ 

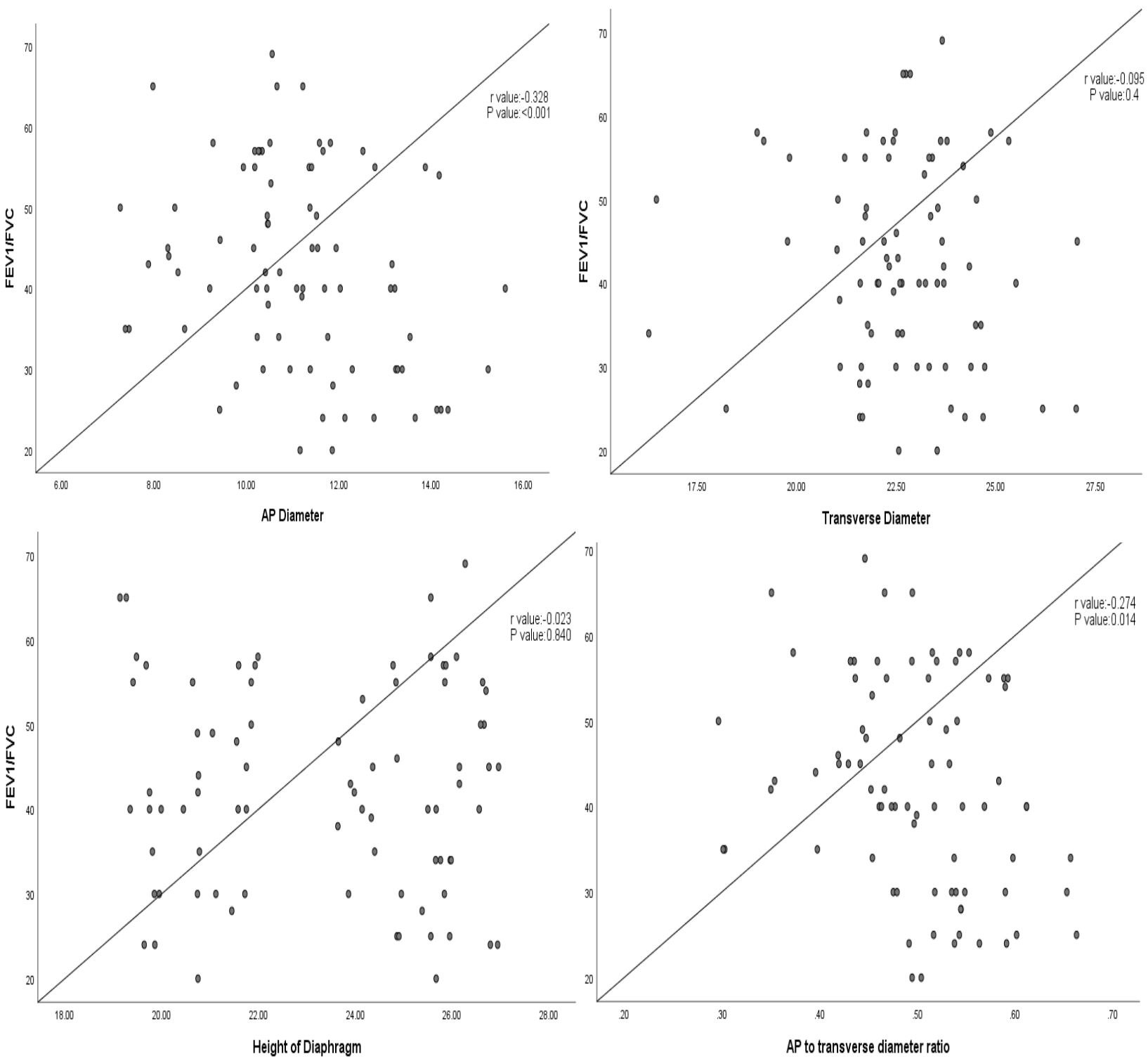

Fig. 2: Scatter plot for Correlation between Thoracic wall dimensions and FEV1/FVC (\%) in the study population (N=80) HDI, AP to Transverse diameter ratio across the MMRC $(\mathrm{N}=80)$

The mean of AP Diameter (mm) was $10.7 \pm 1.82$ in MMRC grade II, it was $10.86 \pm 1.77$ in MMRC grade III and it was $12.55 \pm 1.33$ in MMRC grade IV, and difference across MMRC grade was statistically significant (P-value 0.003). The mean of transverse Diameter $(\mathrm{cm})$ was $22.16 \pm 2.26$ in MMRC grade II, it was $22.62 \pm 1.86$ in MMRC grade III and it was $23.16 \pm 1.44$ in MMRC grade IV, and difference across MMRC grade was statistically not significant (P-value 0.329). The mean of Height of Diaphragm (cm) was $23.8 \pm 2.67$ in MMRC grade II, it was $23.12 \pm 2.59$ in MMRC grade III and it was $23.58 \pm$ 2.64 in MMRC grade IV, and difference across MMRC grade was statistically not significant (Pvalue 0.610 ). The mean of AP to transverse diameter ratio was $0.49 \pm 0.08$ in MMRC grade II, it was 0.49 \pm 0.08 in MMRC grade III and it was $0.55 \pm 0.06$ in MMRC grade IV, and difference across MMRC grade was statistically significant (P-value 0.013 ).

Table 4: Comparison of mean AP diameter, transverse diameter,

\begin{tabular}{|c|c|c|c|c|}
\hline \multirow{2}{*}{ Parameter (Mean \pm SD) } & \multicolumn{3}{|c|}{ MMRC } & \multirow{2}{*}{ P-value } \\
\cline { 2 - 4 } & Grade II & Grade III & Grade IV & \\
\hline AP Diameter & $10.7 \pm 1.82$ & $10.86 \pm 1.77$ & $12.55 \pm 1.33$ & 0.003 \\
\hline Transverse Diameter & $22.16 \pm 2.26$ & $22.62 \pm 1.86$ & $23.16 \pm 1.44$ & 0.329 \\
\hline Height of Diaphragm (cm) & $23.8 \pm 2.67$ & $23.12 \pm 2.59$ & $23.58 \pm 2.64$ & 0.610 \\
\hline AP to Transverse diameter ratio & $0.49 \pm 0.08$ & $0.49 \pm 0.08$ & $0.55 \pm 0.06$ & 0.013 \\
\hline
\end{tabular}

\section{DISCUSSION AND CONCLUSION}

COPD is characterized by progressive, irreversible airflow obstruction. In a significant proportion of patients with COPD, reduction in the lung elastic recoil combined with linmitation in expiratory flow over a period of time leads to dynamic hyperinflation. This Increase in lung volume and 
hyperinflation can cause changes in the shape of the thoracic cage in chronic obstructive lung disease patients. Therefore, it has been traditionally accepted that COPD patients exhibit increased thoracic cage dimensions, especially AP diameter, leading to a, "barrel-chest (4, 8-10). Measurement of severity of the disease is essential for epidemiologic and outcomes research on subjects with COPD. The main objective of the assessment of COPD by the physician is to determine the severity of the disease, the degree of limitation of airflow, and the risk of decompensation. According to the 2017 revised GOLD guidelines, spirometry is no longer considered to have a strong correlation with the symptoms and impairment of the patient's health status (3). A total of 80 subjects were included in the final analysis in our study. The majority (60\%) of the subjects had an mMRC dyspnea grading of 3, while $21.25 \%$ had a grade of 2 and $18.75 \%$ had an mMRC grade of 4 .

There was a statistically significant difference in mean AP diameter and AP to Transverse diameter measured across various grades of MMRC while the difference in mean HDI and transverse diameter across the groups was not statistically significant. In the study by Lim SJ et al.,(4), most patients with COPD were categorized as GOLD stage $2(n=36$, $31.3 \%)$ and GOLD stage $3(n=29,25.2 \%)$. Based on the severity of obstruction, $62.5 \%$ had moderate Obstruction in our study while $37.50 \%$ had Severe Obstruction. There was no significant difference between the groups with respect to baseline anthropometric variables such as mean age, gender distribution in our study. However, the mean BMI was higher in subjects with severe obstruction compared to subjects with moderate obstruction. The diaphragm in COPD patients tends to be shortened due to pulmonary hyperinflation resulting in a shortened apposition zone leading to ineffective contractions because of reduced piston-like motions of the diaphragm. In addition, pulmonary hyperinflation increases the use of the accessory respiratory muscles. The respiratory muscle movements might be reduced by an excessive overlap of actin-myosin filaments. A relationship between increased thoracic cage diameter in COPD patients and lung volume was not documented in the study by Lim SJ et al., (4). They also reported that the ratio of $\mathrm{AP} /$ transverse diameter of the thoracic cage was also significantly greater in patients with COPD compared with normal controls $(0.66 \pm 0.061$ vs. $0.61 \pm 0.86 ; \mathrm{p}=0.002$ ). In our study, the mean AP to transverse diameter ratio was $0.47 \pm 0.08$ in Moderate Obstruction while it was $0.55 \pm 0.05$ in Severe Obstruction and this difference was statistically significant. Several studies have reported changes in thoracic cage diameter according to lung volume. Salito et al., (9) measured rib-cage diameter in five patients with severe emphysema using CT. They reported that the AP diameter of the rib-cage was greater in COPD patients than in controls according to RV, but not Total Lung Capacity (TLC). Lung volume reduction surgery also may reduce AP rib-cage diameter in patients with severe COPD (11). Wang et al., (12) in their study observed that Pulmonary function results, particularly Diffusing Capacity of the Lung for Carbon Monoxide (DLCO) and Residual Volume (RV)/TLC, were primarily affected by the \% Low-Attenuation Volume (LAV) of the upper lobes. Quantitative Computerised Tomography (CT) scan measurements of emphysema provide a morphological method to investigate lung function impairment in patients with chronic obstructive pulmonary disease. Airway involvement and parenchymal attenuation parameters are independent predictors of pulmonary function in patients with mild COPD, whereas parenchymal attenuation parameters are dominant independent predictors of pulmonary function in patients with severe COPD (5). Sileikiene et al., (13) in their study observed that except for the right middle lobe and the right upper lobe, respectively, all the quantitative CT measurements showed weak to moderate negative correlations with diffusing capacity (DLco) $(\mathrm{r}=-$ 0.35 to $-0.61, \mathrm{p}<0.05)$ and weak positive correlations with TLC ( $\mathrm{r}=0.34$ to $0.44, \mathrm{p}<0.05)$. Group analysis indicated that LAV-950 HU (\%) values of both lungs, right lung, left lung, and each lobe, except for right middle lobe, were increased in patients with GOLD stages 3 and 4 of COPD compared to GOLD stages 1 and 2 ( $p<0.05$ ). Pathological changes in COPD are observed in central airways, small airways, and alveolar space. Airway and parenchymal attenuation parameters are independent predictors of pulmonary function in patients with mild COPD, whereas parenchymal attenuation parameters are dominant independent predictors of pulmonary function in patients with severe COPD (5).

COPD may bring changes in the shape of the thoracic cage by increasing the lung volumes. The ratio of average AP and transverse diameter was significantly greater in COPD patients than in controls in the study by Lim et al., (4) BMI was also associated with increased AP diameter in these patients. In COPD patients in their study, the AP diameter of the thoracic cage was positively correlated with BMI and 6-minute walk test distance $(\mathrm{r}=0.395, \mathrm{p}<0.001$ and $\mathrm{r}=0.238, \mathrm{p}=0.028$ ) and negatively correlated with increasing age $(\mathrm{r}=-0.231, \mathrm{p}=0.034)$. Multiple regression analyses in their study revealed an independent correlation only between BMI and increased ratio of $\mathrm{AP} /$ transverse diameter of the thoracic cage $(\mathrm{p}<0.001)$.

In our study, there was a weak positive correlation FVC (L) and HDI, for FEV1/FVC (\%) and HDI there was a weak negative correlation and both were statistically not significant. There was a weak 
negative statistically significant correlation between FVC (L) and AP diameter, FVC (L) and AP transverse diameter ratio, FEV1/FVC $(\%)$ and AP diameter, FEV1/FVC (\%) and AP transverse diameter ratio. There was a weak negative statistically not a significant correlation between FVC (L) and transverse Diameter, FEV1/FVC (\%) and transverse Diameter. Huang et al., (14) in their study observed that a modest negative correlation existed between FEV1\% predicted and mMRC scale and that a significant proportion of subjects with COPD with each scale of mMRC had health status impairment (14). There is a need for large-scale multicentric studies in the Indian population to make strong clinical practice recommendations to use thoracic wall dimensions as early and easily accessible markers of severity of COPD. Munari et al., (15) in their study observed that mMRC was able to predict the Physical Activities of Daily Living alone (time active, $\mathrm{r} 2=0.16$; time sedentary, $\mathrm{r} 2=$ 0.12 ; time $\geq 3$ METs, $r 2=0.12$ ) and was associated with lung function.

The strength of our study lies in the fact that there are no similar published well designed studies in the Indian literature to the best of our knowledge. As the data from other well developed countries may not be the same in Indian population, our study is the first of the kind in our area which can go a long way in paving the way for future clinical recommendations.

The generalizability of our study findings is limited, as the study has been conducted in a single Centre. This study was only a cross-sectional study. The lack of statistical significance of many of the differences between the study groups may be attributed to the smaller sample size. The role of potential confounding by key variables like the presence of comorbidities could not be assessed due to a smaller sample size of the study.

There was a significant moderate negative correlation between the height of the diaphragm and severity of COPD (FVC, FEV1/FVC \%) in this study. In COPD, the mechanical deformation leads to difficulty in breathing because of reduction in the lung capacity of patients. Hence, there is a role for early and aggressive treatment in patients with COPD. There is a need for large-scale multicentric studies on the subject, to enhance the quality of available evidence on the Indian population. Until such quality evidence is awaited, it is difficult to make any strong clinical practice recommendations.

\section{ACKNOLEDGEMENT}

We acknowledge the technical support in data entry, analysis and manuscript editing by "Evidencian Research Associates."

\section{CONFLICT OF INTEREST}

The authors declare no conflicts of interest.

\section{REFERENCES}

1. Angelis, N., Porpodis, K., Zarogoulidis, P., Spyratos, D., Kioumis, I., Papaiwannou, A., et al., Airway inflammation in chronic obstructive pulmonary disease. J Thorac Dis. 2014; 6(Suppl 1): S167-72.

2. Singh, D., Agusti, A., Anzueto, A., Barnes, P. J., Bourbeau, J., Celli, B. R., et al., Global Strategy for the Diagnosis, Management, and Prevention of Chronic Obstructive Lung Disease: the GOLD science committee report 2019. Eur Respir J. 2019; 53(5): 1900164.

3. Patel, A. R., Patel, A. R., Singh, S., Singh, S., Khawaja, I. Global Initiative for Chronic Obstructive Lung Disease: The Changes Made. Cureus. 2019; 11(6): e4985.

4. Lim, S. J., Kim, J. Y., Lee, S. J., Lee, G. D., Cho, Y. J., Jeong, Y. Y., et al., Altered Thoracic Cage Dimensions in Patients with Chronic Obstructive Pulmonary Disease. Tuberc Respir Dis (Seoul). 2018; 81(2): 123-31.

5. Koo, H. J., Lee, S. M., Seo, J. B., Lee, S. M., Kim, N., Oh, S. Y., et al., Prediction of Pulmonary Function in Patients with Chronic Obstructive Pulmonary Disease: Correlation with Quantitative CT Parameters. Korean J Radiol. 2019; 20(4): 683-92.

6. Kang, J. I., Jeong, D. K., Choi, H. Correlation between diaphragm thickness and respiratory synergist muscle activity according to severity of chronic obstructive pulmonary disease. J Phys Ther Sci. 2018; 30(1): 150-3.

7. Hsu, K. Y., Lin, J. R., Lin, M. S., Chen, W., Chen, Y. J., Yan, Y. H. The modified Medical Research Council dyspnoea scale is a good indicator of health-related quality of life in patients with chronic obstructive pulmonary disease. Singapore Med J. 2013; 54(6): 321-7.

8. Sarkar, M., Bhardwaz, R., Madabhavi, I., Modi, M. Physical signs in patients with chronic obstructive pulmonary disease. Lung India. 2019; 36(1): 38-47.

9. Salito, C., Luoni, E., Aliverti, A. Alterations of diaphragm and rib cage morphometry in severe COPD patients by CT analysis. Conf Proc IEEE Eng Med Biol Soc. 2015; 2015: 6390-3.

10. Zhang, D., Guan, Y., Fan, L., Xia, Y., Liu, S. Y. [Quantitative analysis of emphysema and air trapping at inspiratory and expiratory phase multi-slice spiral CT scan in smokers: correlation with pulmonary function test]. Zhonghua Yi Xue Za Zhi. 2018; 98(19): 1467-73.

11. Lando, Y., Boiselle, P., Shade, D., Travaline, J. M., Furukawa, S., Criner, G. J. Effect of lung volume reduction surgery on bony thorax configuration in severe COPD. Chest. 1999; 116(1): 30-9.

12. Wang, G., Wang, L., Ma, Z., Zhang, C., Deng, K. Quantitative emphysema assessment of pulmonary function impairment by computed tomography in chronic obstructive pulmonary disease. J Comput Assist Tomogr. 2015; 39(2): $171-5$.

13. Sileikiene, V., Urbonas, M., Mataciunas, M., Norkuniene, J. Relationships between pulmonary function test parameters and quantitative computed tomography measurements of emphysema in subjects with chronic obstructive pulmonary disease. Acta Med Litu. 2017; 24(4): 209-18.

14. Huang, WC., Wu, M. F., Chen, H. C., Hsu, J. Y., TOLD Group. Features of COPD patients by comparing CAT with mMRC: a retrospective, cross-sectional study. NPJ Prim Care Respir Med. 2015; 25: 15063.

15. Munari, A. B., Gulart, A. A., Dos Santos, K., Venancio, R. S., Karloh, M., Mayer, A. F. Modified Medical Research Council Dyspnea Scale in GOLD Classification Better Reflects Physical Activities of Daily Living. Respir Care. 2018; 63(1): 77-85. 